\title{
EL ASBESTO COMO ELEMENTO PERJUDICIAL EN EL SER HUMANO Y DE IMPACTO AMBIENTAL NEGATIVO A NIVEL MUNDIAL
}

\section{THE ASBESTOS AS A HARMFUL ELEMENT IN THE HUMAN AND OF A WORLDWIDE NEGATIVE ENVIRONMENTAL IMPACT}

\author{
Humberto Peña Rivas ${ }^{1}$ \\ hcpr100271@gmail.com. \\ Emanuel Bohórquez Armijos ${ }^{1}$ \\ ema bohorquez@hotmail.com \\ ${ }^{1}$ Universidad Estatal Península de Santa Elena, Ecuador
}

\section{RESUMEN}

El asbesto es un grupo de minerales no metálicos fibrosos, compuestos de silicatos de doble cadena que poseen gran resistencia a la tensión y la degradación química y conductividad térmica baja. A pesar de la evidencia experimental y poblacional de que los manifestados minerales son agentes cancerígenos y reconocidos por la Organización Mundial de la Salud, aún continúan usándose, en muchos países, de Europa como América Latina su uso continúa, a costa de la salud de los trabajadores, de las familias, ya que el uso de este material impera desde las escuelas, complejos familiares de bajo costo e incluso en los hospitales, lo que se ha convertido en un problema mundial por el desarrollo de enfermedades asociadas a estos minerales en individuos expuestos. El presente artículo se analiza cómo la salud y los daños al medio ambiente se ven afectados, por el uso del asbesto, las enfermedades asociadas a él y el comportamiento mundial frente a esta problemática; además, se plantea la conveniencia de concienciar a la comunidad en general y a los gobernantes sobre lo perjudicial del asbesto y el grave efecto que causa a los individuos expuestos. Finalmente, La nula o muy limitada información y vigilancia sobre el Asbesto y sus riesgos por parte de los Gobiernos, no obstante que la legislación de todos los países, desde hace más de 3 décadas, considera esta sustancia como causante de enfermedad profesional, hace que el asbesto sea el enemigo silencioso del ser humano y del medio ambiente.

Palabras clave: Asbestos; prohibición de su uso de asbestos; Impacto ambiental

\begin{abstract}
Asbestos is a group of non-metallic fibrous minerals, composed of double-stranded silicates that possess high tensile strength and chemical degradation and low thermal conductivity. In spite of the experimental and population evidence that the manifest minerals are carcinogenic agents and recognized by the World Health Organization, in many countries, Europe continues to be used as Latin America its continuous use, at the expense of the health of the Workers, families, since the use of this material prevails from schools, low-cost family complexes and even in hospitals, which has become a global problem for the development of diseases associated with these minerals In exposed individuals. This article analyzes how health and environmental damage are affected by the use of asbestos, the diseases associated with it and the world behavior in front of this problem; In addition, the advisability of raising the awareness of the community in general and the rulers about the harmful effects of asbestos and the serious effect it causes on exposed individuals is raised. Finally, the lack of or very limited information and surveillance on Asbestos and its risks by governments, despite the fact that the legislation of all countries, for more than 3 decades, considers this substance to be the cause of occupational disease, Asbestos is the silent enemy of the human being and the environment.
\end{abstract}

Keywords: Asbestos; Prohibition of their use of asbestos; Environmental impact.

Recibido: 21de febrero de 2017

Aceptado: 5 de julio de 2017

Publicado: 31 de julio de 2017 


\section{Introducción}

Desde tiempos remotos, el hombre en su constante búsqueda por satisfacer sus medios de subsistir, de procurarse alimentos, vestido, vivienda y comodidades; ha utilizado herramientas y métodos que aun siendo perjudiciales para él mismo, no ha dejado de utilizar sin crear conciencia del perjuicio que acarrea para sí mismo y su incidencia negativa en el impacto ambiental.

El uso del asbesto se remonta a 1920, cuando incitados por las penurias de la postguerra, comienzan los estudios mineros sobre el asbesto, a fin de contemplar el uso que posteriormente pudiera tener; es a partir de 1956 cuando la Dirección Nacional de Fabricaciones Militares emite un informe de Inspección mineraindustrial de los yacimientos de asbesto que existían en un país donde se desconocían los riesgo de impacto ambiental y de salud que conllevaban la comercialización y uso del asbesto.

El asbesto pertenece a un grupo de minerales no metálicos fibrosos, compuestos de silicatos de doble cadena que poseen gran resistencia a la tensión, degradación química y conductividad térmica baja. A pesar de la evidencia experimental y poblacional de que dichos minerales son agentes cancerígenos y de su reconocimiento como tales por la Organización Mundial de la Salud, aún se los sigue usando en muchos países.

Asimismo, se ha documentado ampliamente el daño ocasionado en humanos por la exposición a asbesto: enfermedades pulmonares como la asbestosis (una forma de fibrosis pulmonar), placas, engrosamientos y derrames pleurales, y daños del ADN que llevan al desarrollo de cáncer de laringe y pulmón y de mesotelioma ${ }^{1}$.

El efecto perjudicial que produce el asbesto para el ser humano, y el impacto ambiental perjudicial que genera, hace que esta problemática se convierta en un interés común que compete a la humanidad.

El presente estudio analiza desde una perspectiva teórica la utilización indiscriminada del asbesto y cómo repercute el impacto ambiental perjudicial para el ser humano. Muchos son los estudios realizados en referencia a este material y el daño que produce en el ser humano; Sin embargo, su uso es extenso en diversas industrias, sin tomar en cuenta los daños irreversibles que puede ocasionar a la salud y al medio ambiente.

\section{Asbesto}

Asbesto es el nombre usado para referirse a un grupo de minerales no metálicos, fibrosos, compuestos por silicatos de doble cadena; en algunos países también usan el nombre amianto para referirse a este grupo de minerales compuesto por crocidolita, amosita, tremolita, antofilita, actinolita y crisotilo.

Numerosos son los usos que se le ha dado a este material a nivel mundial desde finales del siglo $X I X^{3}$; se ha aplicado en industrias de la construcción y en edificación para reforzar el cemento y los plásticos, así como aislante, en material para techos, material incombustible y para absorber el sonido. Asimismo la industria automotriz usa el asbesto en las zapatas de los frenos y en los discos de embragues de vehículos. El asbesto ha sido utilizado en losas de techos y pisos; pinturas, revestimientos adhesivos, y en algunos lápices de colores que contienen talco, entre otros.

Debido a su composición química, los minerales del asbesto son silicatos, que contienen átomos de silicio y de oxígeno en su estructura molecular. Los minerales de asbesto se dividen en dos grupos principales: asbesto serpentina y asbesto anfibólico. El asbesto serpentina incluye mineral crisotilo, el cual posee fibras largas, rizadas, que se pueden entrelazar. El asbesto crisotilo es el que se ha usado con mayor frecuencia en aplicaciones comerciales.

Ya en los años 70 la Comisión de Seguridad de los Productos para el Consumidor de EE.UU., (U.S. Consumer Product Safety Commission, CPSC) realizó la prohibición del uso del asbesto en los compuestos para la reparación de tablaroca o cartón-yeso y en chimeneas de gas porque las fibras de estos productos podían escaparse al ambiente durante el uso. Posteriormente otros fabricantes suspendieron el uso de asbesto en secadores de pelo eléctrico de forma voluntaria.

Es a partir del 1989 que la Oficina de Protección Ambiental de los Estados Unidos (U:S: Environmental Protection Agency ${ }^{3}$, EPA), prohíbe todo uso nuevo del asbesto; sin embargo todo uso establecido antes de 1989 prevalece. De igual forma estableció normas que requieren que las escuelas sean inspeccionadas en su infraestructura por la presencia de asbesto dañado y para eliminar o reducir la exposición de los ocupantes mediante el retiro o el sellado del asbesto ${ }^{3}$. Actualmente, los diversos estudios realizados a nivel mundial que muestran la gran preocupación generalizada sobre los peligros que representa el uso de asbesto para la salud, 
han llevado a una reducción importante en el uso anual del asbesto en los Estados Unidos.

Para las Instituciones como El Departamento de Salud y Servicios Humanos de los Estados Unidos, la Oficina de Protección Ambiental y la Oficina Internacional para la Investigación del Cáncer, el asbesto ha sido clasificado como una sustancia que causa cáncer; según las investigaciones, la exposición al asbesto puede incrementar el riesgo de cáncer de pulmón y de mesotelioma (cáncer poco común del revestimiento delgado del pecho y del abdomen). Aunque es un cáncer de poca frecuencia, el mesotelioma es el tipo de cáncer asociado más comúnmente con la exposición al asbesto. Otras investigaciones sugieren que existe una relación entre la exposición de asbesto y el cáncer colorrectal y el cáncer gastrointestinal, así como un riesgo mayor de padecer cáncer de garganta, de riñón, esófago y de vesícula biliar. Sin embargo no existe evidencia definitiva. Asimismo, la exposición al asbesto puede aumentar el riesgo de asbestosis (enfermedad inflamatoria que afecta los pulmones y causa dificultad para respirar, tos, y daño permanente a los pulmones) $)^{3}$.

Pero en realidad ¿quién posee el riesgo de padecer una enfermedad relacionada con el asbesto?; Todo el mundo, está expuesto en algún momento de su vida; en el aire, en el agua $y$ en la tierra, se pueden encontrar concentraciones bajas de asbesto; sin embargo no todas las personas suelen enfermarse posterior a la exposición ${ }^{2}$. Las personas que están expuestas de forma continua al asbesto, por lo general en su sitio de trabajo o por contacto directo con el material, son susceptibles de enfermarse. Aunque es evidente que a mayor exposición y tiempo de exposición, mayor será el riesgo para la salud. Generalmente, las personas que presentan enfermedades relacionadas con el asbesto, no muestran signos de la enfermedad por largo tiempo, posterior a la primera exposición; puede tardar años de 10 a 40 años o más, para que aparezcan los síntomas de una enfermedad relacionada con el asbesto ${ }^{2}$.

La exposición al asbesto ocurre por inhalación de fibras dispersas en el aire y puede ser de tres tipos: profesional (personas que manipulan el asbesto o que laboran en sitios de explotación o uso del mismo); doméstica (personas convivientes con trabajadores expuestos al asbesto; también las que viven en casas o edificaciones construidas con materiales a base del mismo); o ambiental (personas que viven o han vivido en la proximidad de sitios que utilizan el asbesto). En la actualidad, alrededor de 125 millones de personas en el mundo están expuestas al asbesto en el trabajo y se calcula que todos los años mueren como mínimo 90.000 personas de cáncer de pulmón, mesotelioma y asbestosis debidos a la exposición al amianto por motivos profesionales ${ }^{4-6}$.

A tal respecto, según datos publicados por la Organización Mundial de la Salud (OMS), en el mundo mueren 318.000 personas al año por enfermedad pulmonar obstructiva crónica (EPOC) asociada a agentes ocupacionales ${ }^{13}, y$ se calcula que el asbesto es el causante de aproximadamente la mitad de las muertes por cáncer profesional ${ }^{14}$. Las características físicas y químicas del asbesto hacen que sea un material muy utilizado en la industria en todo el mundo, lo que, sumado a los casos reportados de enfermedades asociadas en los trabajadores expuestos, ha llevado a su clasificación como agente causal de enfermedades ocupacionales. Esta caracterización ha generado un movimiento mundial en contra de su explotación y uso, y hasta la fecha se ha logrado la prohibición en 54 países, y en otros, la reglamentación sobre su empleo, niveles de exposición, riesgo y monitorización; los países en vías de desarrollo son los menos avanzados al respecto, sin embargo, en países, donde aún se explota, transforma y manipula el amianto en la industria y donde solo recientemente se empieza a reglamentar específicamente su uso (2010), se hace necesario optimizar las medidas de control y vigilancia, que deben incluir la monitorización tanto de la exposición actual y futura como de la acumulada hasta el momento por los trabajadores afectados. Una solución a esta necesidad, en función del desarrollo de cáncer por asbesto, es la Monitorización Biológica Genética, un sistema de alerta y detección precoz del inicio de irregularidades celulares en el desarrollo de enfermedades genéticas y cáncer, que puede ser efectivo en el control y vigilancia de los trabajadores expuestos al asbesto.

La reducción de la utilización de asbesto en los países industrializados ha provocado una reorientación de la industria sobre la base de un "doble estándar". En los países industrializados, procesos de sustitución han permitido dejar el asbesto en todas sus aplicaciones. Sin embargo, en los países en vía de desarrollo, el asbesto sigue siendo un recurso no reemplazado.

\section{Explotación del asbesto}

En Europa, el mercado es prácticamente inexistente con la única excepción, notable, de Rusia, que todavía es el primer productor mundial de asbesto. El debate sobre el asbesto ha sido prácticamente inexistente tanto bajo el régimen soviético como después. Las 
instituciones oficiales de la federación de Rusia siguen negando la importancia de los daños sanitarios del asbesto. La ciudad polaca de Szczucin alberga desde 1959 una importante empresa de asbesto-cemento.

En países de América Latina, producto de la globalización y el crecimiento demográfico, se ha implementado el uso del asbesto en complejos habitacionales, escuelas, universidades, hospitales, entre otros; por el bajo costo económico que el material acarrea, en comparación con otros materiales. No obstante, ante tal planteamiento, se presenta la siguiente interrogante: ¿a qué precio se están mitigando las necesidades de la población? Si bien es cierto que el principal controlador de la normativa es el estado; es el estado mismo quien debe velar por el cumplimiento de estas normativas que controlen el uso indiscriminado del asbesto.

A tal efecto, surgen algunas interrogantes como: ¿Qué riesgos para la salud representa la exposición al asbesto?, ¿Existe otro tipo de materiales que puedan sustituir el asbesto?, ¿Están conscientes las personas sobre los riesgos de salud y de impacto ambiental que acarrea la exposición al asbesto? Es probable que las personas estén expuestas al asbesto en su sitio de trabajo, los niños en la escuela, los enfermos en los hospitales; o en sus propios hogares. Si los productos que contienen asbesto se agitan, millones de fibras de asbesto se desprenderán y esparcirán en el ambiente, provocando la inhalación involuntaria de estas fibras y alojándose en los pulmones, logrando permanecer allí por mucho tiempo. A medida que transcurre el tiempo, estas fibras pueden acumularse y crear cicatrices e inflamación lo que puede acarrear severos problemas de salud.

Argentina fue el primer país que en el año 2000 prohibió el uso de anfíboles y en el 2001 el uso y la importación de crisotilo según Resoluciones del Ministerio de Salud 845 de 2000 y 823 de 200117. Asimismo, Chile en 2001, a través del decreto $N^{\circ} 656$ del Ministerio de Salud publicado en el Diario Oficial el 13 de enero de 200118-19, prohíbe la producción, importación, distribución, venta y uso de todo tipo de asbesto y materiales que lo contienen.

De igual manera Uruguay, según Decreto 154 de 2002 publicado en el Diario Oficial N. ${ }^{\circ} 26.001 \mathrm{del}$ 7 de mayo de $2002^{20}$, acogió políticas en el año 2002 al prohibir su fabricación e importación. Honduras en el 2004, prohíbe el uso de productos que contienen crisotilo, antofilita, actinolita, amosita y crocidolita, así como su importación, fabricación, distribución, comercialización, transporte y almacenamiento, excluyendo a los aislamientos térmicos 0 eléctricos de electrodomésticos, equipos electrónicos y equipos de protección personal contra incendios.

Cabe destacar que actualmente solo países como Argentina, Chile, Uruguay y Honduras, han establecido leyes de prohibición del uso de asbesto, en otros países han determinado restricciones con respecto a la producción, y exportación del asbesto.

Como es de destacar Venezuela importó en el año 2004 aproximadamente 3.800 toneladas de asbesto, y en el 2005 se reportaron 12 empresas importadoras con alrededor de 600 trabajadores, de los cuales se calculó un $10 \%$ con exposición al amianto ${ }^{21}$. Respecto a la normatividad sobre el amianto, Venezuela ha definido $0,1 \mathrm{fibra} / \mathrm{ml}$ de aire como la concentración permisible para todas las fibras de asbesto, según norma Covenin 2253-200122, y ha estipulado las medidas mínimas de seguridad e higiene ocupacional durante el transporte, almacenamiento y uso del asbesto mediante la norma Covenin 2251:199823.

En Colombia, el consumo de asbesto durante el año 2010 fue de 12.312,63 toneladas métricas según los datos publicados por el USG ${ }^{24}$. De acuerdo con el Ministerio de la Protección Social, en el país solo existe una explotación de asbesto crisotilo, con producción aproximada de 9.000 toneladas anuales en los últimos años y de 270.000 toneladas anuales de asbesto-cemento (10\% asbesto + $90 \%$ cemento) registrada en la década de los años $80^{25}$. No se tienen datos exactos de las otras actividades económicas en las que hay exposición al asbesto, pero el grupo de estudio que realizó junto con el Ministerio el Plan Nacional para la Prevención de la Silicosis, la Neumoconiosis del Minero del Carbón y la Asbestosis 2010-2030, logró detectar mediante encuestas a las empresas aseguradoras de riesgos profesionales (ARP), 256 empresas que desarrollan 25 actividades económicas con utilización de asbesto, en las cuales se calculó que el $7 \%$ de los trabajadores (688 de 15.170) están expuestos.

Lamentablemente, estos datos no reflejan la realidad del país, pues a pesar de los esfuerzos del Gobierno por establecer un diagnóstico veraz sobre la exposición al asbesto, fue cuestionable la respuesta por parte de los sectores productivo, de salud y de seguridad social, sumándose a esto los subregistros derivados del trabajo informal, en el que se incluye una gran proporción del sector automotor que manipula material del fricción.

La normatividad colombiana se limita al manejo seguro del asbesto, sin que se haya logrado su prohibición; la sentencia C-493 de 1998 de la 
Corte Constitucional de Colombia indica la aceptación del convenio internacional Protección de los Riesgos a la Salud por la Exposición al Asbesto, el cual, según la sentencia, tiene por objeto "prescribir las medidas que habrán de adoptarse para prevenir y controlar los riesgos para la salud debidos a la exposición profesional al asbesto y para proteger a los trabajadores contra tales riesgos" 26 . Ese mismo año, el Gobierno aprobó el Convenio 162 de la OIT mediante la Ley 436 y posteriormente, en 2001, el Ministerio de Trabajo y Seguridad Social emitió la Resolución 00935, por medio de la cual se conforma la Comisión Nacional de Salud Ocupacional del Sector Asbesto. En el año 2010 el Gobierno Nacional publicó el Plan Nacional para la Prevención de la Silicosis, la Neumoconiosis del Minero del Carbón y la Asbestosis 2010-2030. Aunque la normatividad existente en Colombia establece las pautas para la protección frente al asbesto y el seguimiento sanitario del personal expuesto, no existen estudios poblacionales de los casos de enfermedades ocupacionales asociadas al asbesto que den un dato, por lo menos aproximado, de su prevalencia e incidencia.

Alrededor de 25 países producen asbesto y 85 fabrican materiales que lo contienen ${ }^{29}$. En Europa Occidental, Escandinavia, América del Norte y Australia la fabricación y uso de productos de asbesto llegó a su máximo en la década de los años 70; actualmente, los productores más grandes son Rusia, China, Brasil, Kazajistán y Canadá ${ }^{30-31}$.

Esta postura permite señalar además, que de acuerdo al manejo y uso de asbesto a nivel mundial y a la escasa importancia que algunos países han otorgado a esta problemática, en un futuro no muy lejano se generará una crisis a nivel mundial con un impacto ambiental perjudicial para la humanidad. Las grandes empresas en su constante búsqueda de economizar sus recursos y abaratar los costos de fabricación, utilizan el asbesto y no crean conciencia del daño que se hace a las personas $y$ al medio ambiente.

Finalmente, La nula o muy limitada información y vigilancia sobre el Asbesto y sus riesgos por parte de los Gobiernos, no obstante que la legislación de todos los países, desde hace más de tres décadas, considera esta sustancia como causante de enfermedad profesional, hace que el asbesto sea el enemigo silencioso del ser humano y del medio ambiente.

\section{Conclusión}

Ocultar los riesgos del Asbesto y desinformar acerca de ellos son los rasgos centrales de la gestión empresarial, es por ello que la desinformación, propiciada por las industrias y empresas que emplean Asbesto acerca de sus peligros para la salud y la vida, hace que el asbesto se procure como un enemigo sigiloso. La nula o muy limitada capacidad de inspección por parte de los gobiernos sobre el cumplimiento de la legislación sobre el Asbesto.

En tal sentido, esta problemática emerge como determinante en el inconveniente que impide hacer visible el problema en los países que aún mantienen el manejo y uso de asbesto y es facilitadora de las políticas de uso regulado y de control, debido a las cuales el desconocimiento de los riesgos del Asbesto por parte de los trabajadores, y del ciudadano común acerca de las consecuencias de este material cancerígeno que pone en riesgo su salud, su vida y al medio ambiente.

Es preocupante observar que a pesar de las evidencias mundiales sobre los riesgos para la salud generados por el asbesto aún haya países que sigan usándolo, lo que ha llevado a que las enfermedades producidas por la exposición al asbesto, se hayan convertido en un problema de grandes dimensiones que es necesario abordar de manera inmediata. En algunos países como Colombia, en concordancia con la recomendación de la OMS de hacer planes nacionales para eliminar las enfermedades asociadas al asbesto, solo se limita a abordar el manejo del asbesto de una manera "segura" sin que se vislumbre su prohibición, que es considerada por la OMS como el primer paso para lograr la eliminación de las enfermedades relacionadas con el amianto.

Es importante continuar la con incesante labor de concienciar a los ciudadanos, a las empresas, pero sobre todo a los gobernantes, ya que de ello dependerá la preservación de la vida del ser humano y del medio ambiente.

Finalmente, es importante destacar que dependerá de la actitud asumida por los gobernantes de cada país, realizar las respectivas prohibiciones, y prevención en materia del uso de este material. Asimismo, la creación de valores en cuanto a la concienciación de los individuos en el trabajo, en las escuelas, en materia de conocimiento del asbesto.

\section{Bibliografía}

1. Agency for Toxic Substances and Disease Registry. What Is Asbestos? Retrieved April 
10 ,

2009

from:

http://www.atsdr.cdc.gov/asbestos/more abo ut asbestos/what is asbestos.

2. Agency for Toxic Substances and Disease Registry. Toxicological Profile for Asbestos. September 2001 Retrieved April 10, 2009, from:

http://www.atsdr.cdc.gov/toxprofiles/tp61.pdf.

3. National Toxicology Program. Asbestos. In: Report Carcinogens. Eleventh Edition. U.S. Department of Healt and Human Services, Public Health Service, National Toxicology Program, 2005.

4. Ullrich RL. Etiology of cáncer: Physical factors. In: DeVita VT Jr., Hellman S, Rosenberg SA, editors. Cancer: Principles and Practice of Oncology. Vol. 1 and 27 th ed. Philadelphia: Lippincott Williams and Wilkins, 2004.

5. U.S. Geological Survey. Mineral Commodity Summaries 2006: Asbestos. Retrieved April 10, 2009, from: http://minerals.usgs.gov/mineral/pubs/mcs/20 06/mcs2006.pdf.

6. Agency for Toxic Substances and Disease Registry. Asbestos: Health Effects. Retrieved April 10, 2009, from:

http://www.atsdr.cdc.gov/asbestos/asbestos/ health effects/index.html.

7. U.S. Environmental Protection Agency. Health Effects. Assessment foor Asbestos. September 1984. EPA/540/1-86/049 (NTIS PB86134608). Retrieved April 10, 2009, from: http://cfpub.epa.gov/ncea/cfm/recordisplay.cf m?deid=40602.

8. International Agency for Research on Cancer. Asbestos. IARC Monographs on the Evaluation of Carcinogenic Risks to Humans, vol 14. Lyon, France. Retrieved April 10, 2009 ,

from:

http://monographs.iarc.fr/ENG/Monographs/v ol14/volume14.pdf.Notificaciondesalida

11.Driscoll T, Nelson DI, Steenland K, Leigh J, Concha-Barrientos M, Fingerhut M, et al. The global burdenof disease due to occupational carcinogens. Am J IndMed. 2005 Dec; 48(6):419-31.

12.Driscoll T, Nelson DI, Steenland K, Leigh J, Concha-Barrientos M, Fingerhut $M$, et al. The global burdenof non-malignant respiratory disease due to occupationalairborne exposures. Am J Ind Med. 2005Dec; 48(6):432-45.

13. Concha-Barrientos $D$, Imel Nelson $D$, Driscoll $T$, Steenland NK, Punnett L, Fingerhut MA, et al. Selected occupational risk factors. In: Ezzati M, Lopez A, Rodgers A, Murray C, editors. Comp. Quantif. Heal. Risks Glob. Reg. Burd. Dis. Attrib. to Sel. major risk factors. Geneva: WHO; 2004. p. 1651-801.

14.Organización Mundial de la Salud. Eliminación delas enfermedades relacionadas con el amianto. Ginebra: Organización Mundial de la Salud; 2006. p. 4.

15. Observatorio de la Salud Pública. El amianto y la salud. Barcelona; 2007. p. 4.

16. Valverde M, Rojas E. Environmental and occupational biomonitoring using the Comet assay. Mutat Res. 2009; 681(1):93-109.

17. Asbesto o Amianto, un elementos peligroso [Internet]. Not. Tecnológico Sem. No113. 2008 [cited 2013 Apr 15]. p. 2. Available from: http://www.inti.gob.ar/noticiero/noticiero113.h $\mathrm{tm}$

18. Algranti E, Raile V. El Simposio Sobre Asbesto en América Latina, Sao Paulo, 25-27 de Abril de 2006. Cienc. Trab. 2006; 8(21):122-30.

19. República de Chile Ministerio de Salud. Decreto 656 Prohibe el uso del asbesto en productos que indica. D. Of. 2000; (13.01.2001).

20. Republica del Uruguay. Decreto $154 / 002$ Prohibición de Amianto y Asbestos visto. D. Of. 2002; CVIII (28708):245-A

21 Mujica N, Arteta JM. Asbesto en Venezuela. Cienc. Trab. 20008; 10(27):21-4.

22. Comisión Venezolana de Normas Industriales. Norma Venezolana COVENIN 2253:1997: Concentraciones ambientales permisibles de sustancias químicas en lugares de trabajo e índices biológicos de exposición. Gac. Of. Caracas: Fondo Norma; 1998. p. 24.

23. Comisión Venezolana de Normas Industriales. Norma Venezolana COVENIN 2251:1998: Manejo de asbesto. Caracas: Fondo Norma; 1998.

24. Virta R I. Asbestos. In: U.S. Geological Survey Minerals Yearbook, editor. 2011 Miner. Yearb. Washington, D.C.: U.S.G.S.; 2011. p. 8.1-8.6.

25. Colombia Ministerio de Protección Social. Plan nacional para la prevención de la silicosis, la neumoconiosis de los mineros de carbón y la asbestosis 2010 - 2030. Bogotá D.C.: Ministerio de la Protección Social; 2010. p. 141. 
26. Corte Constitucional de Colombia. Sentencia C-493/98: Convenio Internacional Protección de los riesgos a la salud por la exposición al asbesto. Bogotá D.C.: Corte Constitucional de Colombia; 1998. p. 19.

27. Colombia Ministerio de Trabajo y Seguridad Social. Resolución 00935 de 2001: se conforma la comisión nacional de salud ocupacional del sector asbesto. D. Of. $2001 ;(42137)$.

28.Consejo Consultivo Laboral Andino. Por la prohibición del amianto 0 asbesto en la subregión Andina. Lima: Consejo Consultivo Laboral Andino; 2007. p. 28.
29. Nishikawa K, Takahashi K, Karjalainen A, Wen C-P, Furuya S, Hoshuyama T, et al. Recent mortality from pleural mesothelioma, historical patterns of asbestos use, and adoption of bans: a global assessment. Env. Heal. Perspect. 2008 Dec; 116(12):1675-80.

30. Brasil Departamento Nacional de Produção Mineral. Sumário Mineral 2011. Brasília: DNPM/DIPLAM; 2012. p. 128.

31. Virta R I. Asbestos. In: U.S. Geological Survey Minerals Yearbook, editor. 2011 Miner. Yearb. Washington, D.C.: U.S.G.S.; 2011. p. 8.1-8.6. 